\title{
Utilization of the Community Health Worker Assessment and Improvement Matrix to Strengthen Village Health Team Systems in Uganda: A Case for Kitgum District
}

\author{
Babughirana Geoffrey ${ }^{1}$, Tusaasire Joab ${ }^{1}$, Musasizi Benon ${ }^{1}$, Lule Mark ${ }^{1}$, Muhuma Muyima Ceasar ${ }^{2}$, \\ Twinomujuni Edgar ${ }^{1}$
}

${ }^{1}$ World Vision, Kampala, Uganda

${ }^{2}$ Real Medicine Foundation, Yumbe, Uganda

Email address:

gbabughirana@gmail.com (B. Geoffrey), joabt2004@yahoo.co.uk (T. Joab), musasizi.benon1@gmail.com (M. Benon), lulemark62@gmail.com (L. Mark), muhumaceasar@gmail (M. M. Ceasar), twinruk@gmail.com (T. Edgar)

To cite this article:

Babughirana Geoffrey, Tusaasire Joab, Musasizi Benon, Lule Mark, Muhuma Muyima Ceasar, Twinomujuni Edgar. Utilization of the Community Health Worker Assessment and Improvement Matrix to Strengthen Village Health Team Systems in Uganda: A Case for Kitgum District. Science Journal of Public Health. Vol. 5, No. 4, 2017, pp. 275-287. doi: 10.11648/j.sjph.20170504.11

Received: March 26, 2017; Accepted: April 5, 2017; Published: May 5, 2017

\begin{abstract}
In Uganda community empowerment and mobilization for health has been enshrined in national health policies and strategies since 1999. The USAID Health Care Improvement Project developed the CHW AIM Toolkit that includes a guided self-assessment and performance improvement process to help organizations identify program strengths and address gaps for improving functionality. The study was conducted to ascertain the extent to which the utilization of the CHW- AIM strengthened the VHT systems in accordance with the MoH 2010 guidelines. Using the CHW-AIM methodology at the end line assessment, 6 program components scored 3 a best practice of functionality $(1,3,4,6,8$, and 10), 8 program components scored a functional score of $2(2,5,9,11,12,13,14,15)$ and only one component scored a partially functional score of $1(7)$. The District and the Health Facilities should use this VHT Assessment tool periodically to identify areas of weakness and make improvements accordingly.
\end{abstract}

Keywords: CHW-AIM, Village Health Teams, Community Health System's Strengthening,

Village Health Team Functionality

\section{Introduction}

\subsection{Operational Definitions}

The community health worker: This is a person chosen by the community to facilitate the promotion of the health and well-being of all village members through behavioral change models [1]. In Uganda a community health worker (CHW) is equivalent to a village health team (VHT) [2].

CHW AIM: A kit designed to help organizations assess VHT program functionality and improve program performance [3]. Built around a core of 15 components defined as essential for effective programs, CHW AIM includes a guided self-assessment and performance improvement process to help organizations identify program strengths and address gaps. Through discussion and review of current practices, the process assists understanding of best practices, builds consensus about and commitment to change, and provides guidance for improving functionality.

VHT Functionality process: this involves the scoring of the current VHT implementation and status against the $15 \mathrm{CHW}$ AIM program components at the district level. Each of the component is scored ranging from zero (non-functional) to three (best practice). The overall score of the given geographical area constitute its VHT functionality [4].

\subsection{The CHW Functionality, a Global Strategy}

According to WHO, 57 countries from Africa to Asia are facing shortages in their health care workforces, and an estimated 4,250,000 workers are needed to fill the gaps. CHWs have played an important role in linking communities to health services for over 50 years [5]. The importance of 
CHWs in health systems is becoming increasingly recognized as government institutions are unable to train enough health workers to meet their needs while at the same time the health workforce is being depleted by migration, and inadequate infrastructure [9]. By providing basic health tasks, CHWs free up skilled health providers to offer more complex health care.

The emphasis on community support makes assessing the functionality of CHW programs increasingly important [3]. However, evaluating $\mathrm{CHW}$ programs and how individuals delivering services are selected and supported can be difficult, particularly since characteristics, roles, and responsibilities for CHWs can vary vastly. The USAID Health Care Improvement (HCI) Project developed the CHW AIM Toolkit to help organizations assess CHW program functionality and improve program performance. Built around a core of 15 components as explained in table 1 deemed essential for effective programs, CHW AIM includes a guided self-assessment and performance improvement process to help organizations identify program strengths and address gaps [5]. Through discussion and review of current practices, the process assists understanding of best practices, builds consensus about and commitment to change, and provides guidance for improving functionality. The assessment processes focuses on the assessment of the functionality and guide improvement in programs delivering community services, provide action planning and best practices to assist in strengthening programs and identify the location of functional $\mathrm{CHW}$ programs and gaps in coverage [3].

Table 1. The 15 programmatic components of CHW functionality according to the Community Health worker Assessment and Improvement Matrix Toolkit [5].

\begin{tabular}{|c|c|c|}
\hline \multicolumn{3}{|l|}{ Component Definition } \\
\hline $\begin{array}{l}\text { 1. Recruitment: How and from where a } \\
\text { community health worker is identified, } \\
\text { selected, and assigned to a community. }\end{array}$ & $\begin{array}{l}\text { 6. Supervision: Supportive supervision is carried } \\
\text { out regularly to provide feedback, coaching, } \\
\text { problem solving, skill development, and data } \\
\text { review. }\end{array}$ & $\begin{array}{l}\text { 11. Opportunity for Advancement: The possibility for } \\
\text { growth and advancement for a CHW. }\end{array}$ \\
\hline $\begin{array}{l}\text { 2. CHW Role: The alignment, design, and } \\
\text { clarity of role from community, CHW, and } \\
\text { health system perspectives. }\end{array}$ & $\begin{array}{l}\text { 7. Individual Performance Evaluation: Evaluation } \\
\text { to fairly assess work during a set period of time. }\end{array}$ & $\begin{array}{l}\text { 12. Documentation and Information Management: } \\
\text { How CHWs document visits, how data flows to the } \\
\text { health system and back to the community, and how it } \\
\text { is used for service improvement. }\end{array}$ \\
\hline $\begin{array}{l}\text { 3. Initial Training: Training is provided to } \\
\text { the CHW to prepare for his/her role in } \\
\text { service delivery and ensure s/he has the } \\
\text { necessary skills to provide safe and quality } \\
\text { care. }\end{array}$ & $\begin{array}{l}\text { 8. Incentives: A balanced incentive package } \\
\text { includes financial incentives such as salary and } \\
\text { bonuses and non-financial incentives such as } \\
\text { training, recognition, certification, uniforms, } \\
\text { medicines, etc. appropriate to job expectations. }\end{array}$ & $\begin{array}{l}\text { 13. Linkages to Health Systems: How the CHWs and } \\
\text { communities are linked to the larger health system } \\
\text { through involvement in recruitment, training, } \\
\text { incentives, supervision, evaluation, equipment and } \\
\text { supplies, use of data, and referrals. }\end{array}$ \\
\hline $\begin{array}{l}\text { 4. Continuing Training: Ongoing training is } \\
\text { provided to update CHWs on new skills, to } \\
\text { reinforce initial training, and to ensure s/he is } \\
\text { practicing skills learned. }\end{array}$ & $\begin{array}{l}\text { 9. Community Involvement: The role that the } \\
\text { community plays in supporting a CHW. }\end{array}$ & $\begin{array}{l}\text { 14. Program Performance Evaluation: General } \\
\text { program evaluation of performance against targets, } \\
\text { overall program objectives, and indicators carried out } \\
\text { on a regular basis. }\end{array}$ \\
\hline $\begin{array}{l}\text { 5. Equipment and Supplies: The requisite } \\
\text { equipment and supplies are available when } \\
\text { needed to deliver expected services. }\end{array}$ & $\begin{array}{l}\text { 10. Referral System: A process for determining } \\
\text { when a referral is needed, a logistics plan in } \\
\text { place for transport and funds when required, a } \\
\text { process to track and document referrals. }\end{array}$ & $\begin{array}{l}\text { 15. Country Ownership: The extent to which the } \\
\text { ministry of health has policies in place that integrate } \\
\text { and include CHWs in health system planning and } \\
\text { budgeting and provides logistical support to sustain } \\
\text { district, regional and/or national CHW programs. }\end{array}$ \\
\hline
\end{tabular}

Scoring of programmatic components: $0=$ non-functional $1=$ partially functional $2=$ functional $3=$ best practice

\subsection{The VHT Functionality in Uganda}

The MoH established VHTs in 2001 as a means to close the gap in delivery of health services to the households [10]. Strategy and implementation guidelines were developed and distributed to districts to utilize in establishing, training and motivating the VHTs to undertake their activities. A number of districts and partners have established and trained VHTs in their operational areas with and without guidance of $\mathrm{MOH}$ [6]. In some instances, partners have taken up VHTs, provided training on their programmatic areas without the basic training required of VHTs using the MOH VHT training manual and therefore leaving gaps in the operationalization of the VHT strategy.

An assessment commissioned by the $\mathrm{MoH}$ revealed that Uganda has a total of 179,175 VHTs of which Thirty percent of these do not have basic training, $52 \%$ of these had a minimum of ' $\mathrm{O}$ ' Level qualification. This is an indication that actually Uganda has a community work force that is relatively educated to offer health services [6]. The study further revealed that $47 \%$ of the districts had training of trainers (TOTs) conducted in the last 4 years and $91.1 \%$ of the VHTs had had the initial training.

The VHTs are basically trained to play roles including mobilization of communities to access health interventions such as immunization, mosquito nets distribution, fistula services, HIV/AIDS counselling and testing services. VHTs conduct community sensitizations on disease prevention such as hygiene and sanitation practices e.g. hand washing, construction of pit latrines and drinking boiled water, importance of uptake of health services e.g. HIV testing, antenatal care and family planning [11]. They are actively involved in treatment of common ailments such as malaria, diarrhea and promotion of health services including immunization activities, identifying patients suffering from neglected tropical diseases (NTDs) in their communities [12]. VHTs were also involved in distributing contraceptives such as condoms. They are also instrumental in the distribution of drugs mainly deworming tablets and anti- 
malaria in the communities to population groups such as children under five [2].

\subsection{The VHT Functionality Anticipated Role in the Kitgum}

World Vision Uganda has extensive knowledge of and experience with community based implementation models and interventions that were proposed under East Africa maternal newborn and child Health (EAMNCH) project. Examples such as timed and targeted Counseling (ttC), community led total sanitation (CLTS), positive Deviant hearth (PDHearth), Essential Nutrition Action (ENA) through the parent support groups (PSGs), and community conversations using the stepping stones methodologies [7]. All these models were proposed to implement the 7-11 approach that had been developed by World Vision International, based on its many years of community $\mathrm{MNCH}$ project experience [8]. The approach includes 7 crucial interventions for pregnant women and 11 interventions for children, as presented in the Figure 1. Therefore if functioning well, the government initiated VHT concept and structure had a lot of potential. VHTs are the link between the community and the health services and can transfer knowledge and information between the 2 entities. As community members they know the situation on the ground well. They can ease the workload of the health workers by building the community's capacity to prevent diseases, conducting home treatment where possible, and mobilizing community for outreach events and services offered at HCs [13]. They are a relatively cost effective way of providing services as they are volunteers. If VHTs would receive more training in the planned models and follow up support, they could be powerful change agents in their communities. In that case the EAMNCH project would be able to build upon the updated basic and general knowledge that VHTs will be receiving and increase their capacity in specific $\mathrm{MNCH}$ issues, such as ttC. However, even if the targeted VHTs will not yet have received the updated basic VHT training, the
EAMNCH project would facilitate the District to do so and then plan to provide the additional trainings. To be able to identify the capacity of the VHT systems at that time, there was need to conduct a VHT functionality assessment, and keep facilitating these assessments together with the district to ascertain the improvement contributed by World Vision and other partners.

\subsection{Problem Statement}

At the beginning of the EAMNCH project in Kitgum in 2011, the baseline assessment carried out revealed that $63.6 \%$ of pregnant mothers in the catchment area had never been visited by a VHT during the pregnancy period, $27.2 \%$ of the children were still going for immunization after 9 months due to poor mobilization for the service by the VHTs, and that it takes close to $1.6 \mathrm{hrs}$ for a pregnant mother to walk to the health facility for Health education. The VHT mapping in the project area revealed that $67 \%$ of the VHTs in the project site had undergone the basic training using the $\mathrm{MoH} 2010$ strategy, of these only $46.7 \%$ VHTs had the basic equipment for doing community work. $18.7 \%$ of the population said that they had gone to the VHTs for health services, $31.5 \%$ received Hygiene and sanitation related education, with an overall district functionality of $42 \%$ against the 15 program components. Kitgum's poor health indicators, could partly be tackled through constant dialogue with the community members using VHTs. VHTs help bridge the gap that exists between the un-served households and the formal health system. VHTs were established by the $\mathrm{MoH}$ to empower communities to take part in the decisions that affect their health; mobilize communities for health programs, and strengthen the delivery of health services at house-hold level. It was therefore important to institute a VHT functionality improvement implementation framework and use the CHWAIM to improve health service availability and access to households in the project site.
Pregnant Women: 0-9 months

I. Adequate diet

2. Iron/folate supplements

3. Tetanus toxoid (TT) immunisation

4. Malaria prevention, treatment access and intermittent preventive treatment

5. Birth preparedness and healthy timing and spacing of delivery

6. De-worming

7. Access to maternal health service: antenatal care (ANC), post-natal care (PNC), SBAs, prevention of maternalto-child transmission (PMTCT), HIV/tuberculosis (TB)/ sexually transmitted infection (STI) Screening

\section{Children: 0-24 months}

I. Appropriate breast-feeding

2. Essential newborn care

3. Hand washing with soap

4. Appropriate complementary feeding

5. Adequate iron

6. Vitamin A supplementation

7. Oral rehydration therapy (ORT)/zinc

8. Prevention and care seeking for malaria

9. Full immunisation for age

10. Prevention and care seeking for acute respiratory infection (ARI)

II. De-worming (+12 months)

Figure 1. The 7-11 Focus Issues for MNCH in World Vision International community implementation strategy [8]. 


\subsection{Justification of the Problem}

World vision Uganda through its EAMCH project in Kitgum district supported the establishment and capacity building of VHTs in the operational areas with the MOH's guidance. VHTs have operationalized, provided training on programmatic areas with the basic/core training required of VHTs using the MOH VHT training manual and tried to support the other components that promote the functionality of the VHT Program. This support however has not been offered to the whole district but to the sub counties of Lagoro and Mucwini and therefore the need to evaluate and find a comparison between the implementation areas with areas that have not been supported by the CHW-AIM processes apart from conducting the Annual processes for scoring.

\subsection{General Objective}

To ascertain the extent to which the utilization of the $\mathrm{CHW}$ - AIM strengthened the village health team systems. With the following specific objectives:

a) To assess the improvement of the CHW-AIM 15 program component in the EAMNCH project area by 2016.

b) To assess the overall functionality of the VHTs in the project area in comparision to the Kitgum District status at the close of the project in 2016

c) To document the best practices and provide an improvement plan for existing gaps.

\section{Methodology}

\subsection{Location of the Project}

The study was conducted in Kitgum district which is in the northern part of Uganda. It is located between Longitude $320 \mathrm{E}$, and $340 \mathrm{E}$, Latitude $020 \mathrm{~N}$ and $040 \mathrm{~N}$. It is bordered by Lamwo District in the North, The Republic of Southern Sudan in the North East, Kotido District in the East, Pader District in the South and Gulu District in the Northwest. Kitgum district has a total Land Area of 4,042 square Km. The study was carried out in Kitgum because this is the operational area for the EAMNCH project specifically in the sub counties of Mucwini and Lagoro. This was also used as an evaluation mechanism showing the state of the VHT functionality status at the end of $\mathrm{EAMNCH}$ project versus the beginning of the project.

\subsection{CHW-AIM Progressive Processes Throughout the Project}

To utilise the CHW-AIM tool to monitor and facilitate improvement, in the EAMNCH project area and the Kitgum District the project facilitated an initial assessment, of village health team functionality. It involved 100 VHTs from World vision Uganda implementation sub-counties and these were compared with 100 VHTs from sub-counties where there were no implementation processes as yet. Further still at the district level a guided self-assessment and performance improvement process to help the project and the district identify program strengths and address gaps for improving functionality was followed. The process was repeated at the mid-term review process and then at the end-line.

In this study, The CHW-AIM was a participatory selfassessment process and did not focus on individual CHW program performance, but created a forum for management, supervisors, program staff and service providers, including VHTs, to share views on their programs' current status on 15 practices, those views were used to measured functionality and determined system gaps. These were generated by discussion to reach consensus, including the creation of an improvement action plan together.

The participants used two 2 key resources, a program functionality matrix with 15 key components to assess the current status of their program and a service intervention matrix to determine how VHT service delivery aligns with the Ministry of Health 2010 guidelines especially how it strengthened the VHT systems.

\subsubsection{Process Components Taken into Consideration}

The processes integrated five components: facilitation, participants, approach, acknowledging limitations and the scoring of the 15 programmatic components

Facilitation: A trained facilitator, external to World Vision and the Kitgum District local government ran a workshop to guide the planning, implementation and follow up of the assessment.

Participants: The assessment was carried out during a workshop with multiple stakeholders knowledgeable about how the VHT program is managed or supported. One work shop would have 25 attendees comprising of field level managers, district level managers, VHTs, and VHT supervisors.

Approach: The facilitator used a guided self-assessment approach which allows a diverse group of participants to score their own program against fifteen programmatic components that contribute to successes or shortfalls, and four levels of functionality. After the assessment, the participants of the workshop would finalize the review through discussion to generate the results that were used to develop action plans to address the gaps and or weaknesses in performance of the program.

Scoring of Programmatic Components: For each of the 15 components, four levels of functionality were assigned, ranging from non-functional (Level 0 ) to highly functional as defined by suggested best practices (Level 3). These levels were defined as $0=$ nonfunctional; $1=$ partly functional; $2=$ functional; 3 = best practice

\subsubsection{Steps Taken During the Functionality Assessment Process}

a) Preparation

The first step involved data collection from the district sub-counties and analysis (Microsoft Excel 2007 and SPSS were used) and finally preparation of a power point 
presentation to inform the invited participants on scoring process

b) Conducting the Assessment Workshop

Participants were introduced to the tool, process, and expected outcomes. Participants then reviewed the fifteen programmatic components as a group to ensure comprehension and consistency, and then in small groups to reach consensus. After consensus, then ratings (0 to 3 ) for each programmatic component were recorded.

In the workshop, Participants also assessed program interventions, to determine if they were in compliance with the services and subtasks as defined in national guidelines of 2010. For a component to be rated functional (score 3) all subtasks within that service area were found complete. The group also generated Action items to address weak or nonfunctional areas, discussed and documented them into an improvement action plan.

c) Validation:

Scores from the assessment workshop were validated through interviews with VHTs that did not participate in the workshop and also conversations with community members were conducted in both low-performing and high-performing sites. This generated more learning about the implementation context. Based on these semi-structured interviews, workshop scores and actions inconsistent with the information gathered were reviewed and revise. This was more informed by the initial assessment that was conducted on the VHTs themselves.

\subsection{Provision of Follow-Up Support}

This involved validating the results of the workshop, reviewing and revising as necessary the action plan, getting buy-in from others on supporting the plan, developing a process for monitoring the achievement of the actions and planning re-assessments as desired. This was a joint work with the organizations and ministry of health who participated in conducting the assessment to develop common approaches to achieving best practices based on the 15 components. Further still for EAMNCH project specifically, the plan of action provided a basis for investment into the program components that actually needed support at the sub county level.

\subsection{Conceptual Framework}

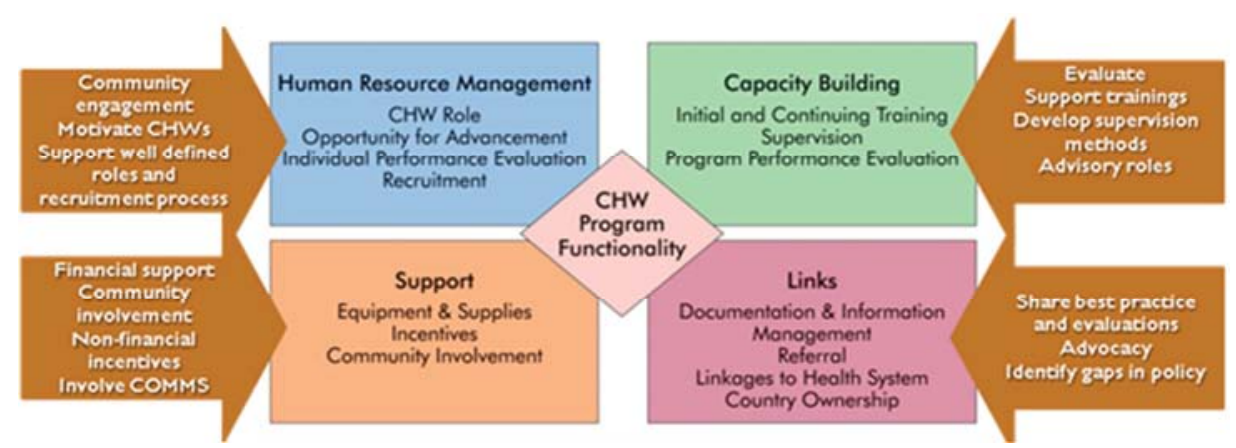

Figure 2. Frame work of the CHW-AIM functionality [14].

A functional CHW program is built around 4 pillars including human resource management, capacity building processes, supporting the $\mathrm{CHW}$ to perform and ensuring linkages to the broader health system. It's these pillars that constitute the 15 program components that the EAMNCH project focused on to build up a functional $\mathrm{CHW}$ program. For the 4 pillars to be effective, the EAMNCH focused on engaging the community to participate and motivate the $\mathrm{CHW}$, there was a deliberate effort to provide for financial and nonfinancial resources, performance of periodic evaluations for the program jointly and ensure the sharing of best practices with other partners and the CHWs themselves. This eventually was anticipated to result into a functional CHW program.

\subsection{Data Management Throughout the Project Life Span}

From the start of the project, to the MTR and eventually the End line assessment, for the information collected from the field with the VHTs, double data entry was carried out to prevent losses of any data. Data from the field was kept under lock and key accessible to the World Vision staff and other members of the research team. Field editing was used to minimize missing data.

\subsubsection{Data Management}

The quantitative data was checked for completeness prior to entry in an electronic database using Statistical Package for Social Scientists (SPSS) Version 20. Qualitative data was transcribed, cleaned, and entered into a master sheet. Under the guidance of the field supervisor/facilitator while trained research assistants captured data, which will securely kept.

\subsubsection{Data Analysis}

Microsoft Excel 2007 for Windows was used as well as SPSS. The responses were coded and entered into an excel data base and for further analysis it was exported into SPSS to avail out-puts in frequency tables, graphs, and relationships among the variables (cross tabs).

\subsection{Ethical Consideration}

The study sought and obtained ethical approval from 
Makerere University School of Public Health Higher Degrees, Research and Ethics committee before it was conducted. In addition, Uganda national council for science and technology (UNCST) also approved the study under number SS 4178 Informed consent was sought from the population interviewed and other participants by signing on the administered consent form.

\subsection{Study Limitation}

The methodology relied on secondary evidence, that is self-reports for assessment, as the information collected. This prevented objective evaluation of individual VHT performance in terms of contribution to coverage, effectiveness or impact of the program. Therefore there is an inherent limitation of the procedure that it did not evaluate the quality of services delivered by individual health workers.

\section{Implementation Results}

\subsection{Village Health Team Mapping}

At the inception of the project, a VHT functionality mapping was done line with the MoH VHT strategy recommendation to ascertain the number of VHT against the Households. The strategy recommends that each VHT is in charge of 30 households [2, 6, and 15). The Mapping revealed that there were 7617 Households in 120 Villages being over seen by 321 VHTs. This simply meant that every VHT was overseeing 23.7 households less than the 30 household's recommendation by the $\mathrm{MoH}$. The district stakeholders therefore recommended that the project facilitates a cropping exercise where some VHTs who were not active and those who were overseeing a few households to be laid off for effective implementation. The project worked with the sub county Health Assistants (Has) and the Villages' leadership to collect feedback about the VHTs ability to work, whether they met the $\mathrm{MoH}$ educational level criteria of reading and writing and how frequent they participate in voluntary work, this information determined the VHTs to be dropped. At the end of the exercise it was recommended that the project facilitates and strengthens 255 VHTs making each VHT to be responsible for 29.8 Households in the project area and therefore in line with the MOH VHT strategy recommendation.

\subsection{Improvement Results from the $15 \mathrm{CHW}-A I M$ Components over the 5 Years of Implementation}

Over the years, the CHW-AIM tool was used to be able to evidently facilitate improvement and quality assurance for the programming through the VHTs. The implementation of the $\mathrm{ttC}$ in the project meant that the $\mathrm{CHWs}$ needed to have the required skills to be able to actually counsel and effect behavioral change in the households [16]. This outcome was heavily dependent on the Functionality of the VHTs and the CHW-AIM was used to measure that, and identify gaps to strengthen the VHT system in the project implementation area.

\subsubsection{Recruitment Process}

This component seeks to find out how and from where a VHT is identified, selected, and assigned to a community. The MoH VHT strategy guidance is that the Community members under the leadership of the Village leadership elects the VHTs to serve the community. The strategy further guides that the members selected are residents of that village and are geographically distributed in different corners of the Village for easy access. Figure 3 (recruitment) shows how this component was rated throughout the project life span. At the baseline it was noted that the component presented a best practice with a score of 3 , but declined to 2.5 in the middle of the project life span because the stakeholder felt that from the results of the 255 VHTs, most of the VHTs are male(145M: 109F) and therefore giving a gender bias mostly towards the implementation of $\mathrm{ttC}$ which focus on the women in the community, further still the stakeholder felt that literacy was not mostly considered since most VHTs could not read and write English well which was a requirement for implementation of $\mathrm{ttC}$ mostly to report on the scheduled visits performed by the VHT and facilitating referral notes. The project therefore invested in mentorship and translated the ttC counselling cards, ttC household register and the referral forms into Acholi and this score at the end of the project assessment rose back to the score of 3 .

\subsubsection{CHW Role}

This component seeks to provide direction at implementation on the alignment, design and clarity of VHT role from the perspective of the community, the VHT themselves, and the entire health system perspectives. Figure 3 (CHW Role) provides the score for this component as almost 2 all through the project life span. The stakeholders agrees that the role and expectations are clear to VHTs as stated in the MoH VHT strategy and this makes the component functional. Inherently to this component even when it began at the functional score of 2 , the project efforts to facilitate the improvement of the score to a best practice of 3 yielded no results as the scoring process at midyear pointed out that the community play no part in defining roles of VHTs, and that the VHTs have high expectations from the community and partners even when they are aware that their services to the community are purely voluntary. Further still at the closure of the project it was visible that updating and discussing roles and task of VHTs at Community level is inadequate and needed strengthening.

\subsubsection{Initial Training}

This component highlights the importance of the training provided to CHWs to prepare for services delivery and ensure they have the necessary skills to provide safe and quality care. The MoH VHT strategy provides a 5 day training that is supposed to be provided to all the VHTs before they set off to facilitate their communities. Further still this is a requirement by the $\mathrm{ttC}$ implementation guidelines for World Vision Uganda that actually the VHTs to roll out ttC are supposed to be VHTs as defined by the Ministry of Health [17]. From the Figure 3 (initial training) at the 
inception of the project, the score for this component was 1 because of the 255 VHTs selected only 150 had been trained in the MoH general strategy giving a $58.8 \%$ of the VHTs.
The remaining VHTs had been selected using the required criteria but not trained in the general strategy.

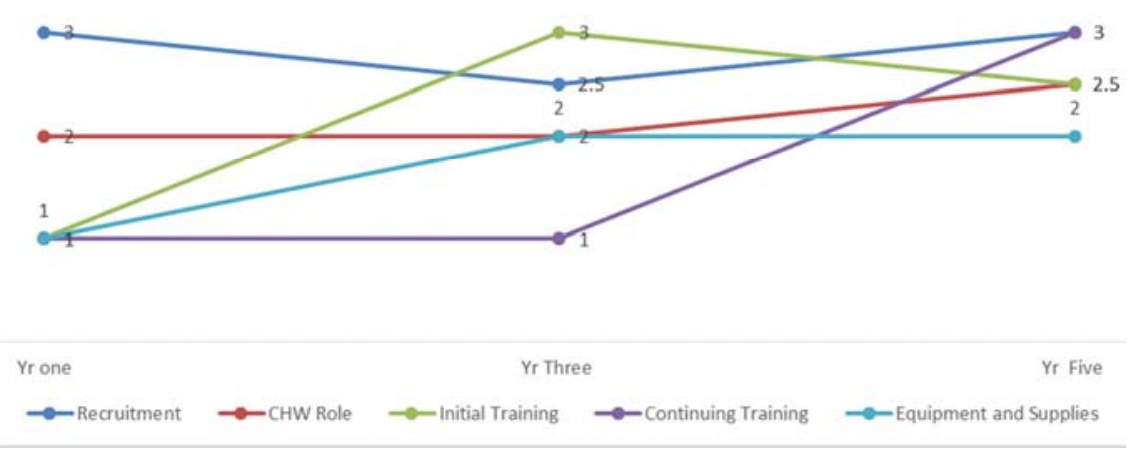

Figure 3. Comparision of the first 5 core components throughout the project cycle.

Because of that recommendation the Project facilitated the training of the remaining VHTs in the general strategy, by the midterm review all the VHTs had been certified as $\mathrm{MoH}$ trained VHTs. However by the closure of the project, the score lost a 0.5 score since the field results showed that $86.7 \%$ of the VHTs actually had been trained. The $13 . \%$ of the VHT who hadn't been trained arose from the fact that by the fourth year of the project some VHTs who were trained had migrated, got married to men in other geographical location and had been replaced by new VHTs that hadn't been trained. The Stakeholders recommended that resources be identified to push the score back to a best practice by training the $13.3 \%$ VHTs that need the initial training mostly if they are to support the continuation of $\mathrm{ttC}$ implementation in the project site.

\subsubsection{On-Going Training}

On-going training to update $\mathrm{CHW}$ on new skills, reinforce initial training, and ensure the $\mathrm{CHW}$ is practicing the skills. The Ministry of Health guidelines of 2010 recommends that the VHTs have to be refreshed in the initial training through meetings and mentorship sessions, but also encourages partners to train the VHTs in any additional training ranging from the integrated community case management (ICCM), community newborn care, maternal and child health, hygiene and sanitation basing on the focus of that implementation partner. Further still the training curriculum should be approved by the $\mathrm{MOH}$ and a training cascade program should be implemented that includes conducting a Training of Trainers at the district level so that the district officials are the ones that actually facilitate the trainings to the VHTs. The EAMNCH project facilitated a number of additional trainings following the above recommendation including the $\mathrm{ttC}$, Community led total sanitation (CLTS), implementation of the PSGs, Stepping stones for Hygiene and sanitation, Kitchen gardening and animal husbandry and facilitated quarterly mentorships in the above. Figure 3(continuing training) shows that at the inception of the project, the core component scored 1 but by the end of the project, it was at 3 which is the best practice. This was as a result of the strategies that the project collaboratively implemented with the district in the project site. The project also benefited from a collaboration with another partner who was implementing iCCM in the project area using the same VHTs.

\subsubsection{Equipment and Supplies}

Required equipment and supplies to deliver expected services including job aids. All necessary supplies, are available with no substantial stock out periods, health facility takes account of CHW needs when ordering their supplies. Supplies are checked and updated regularly to verify expiration dates, quality, and inventory. The $\mathrm{MoH}$ guidelines puts emphasis on the VHT Generic Job aids, the House hold register, the community referral, the participant's manual and the quarterly reporting form that the VHT submits to the VHT supervisor. These should preferably be translated into the local language that the VHT can read and write. Figure 3 (equipment and supplies) shows an improvement from 1 score at the inception of the project to 3 at the end of the project. The EAMNCH through the $\mathrm{MoH}$ facilitated the translation of all the materials into the Acholi language and effected their availability to the 255VHTs in the project area. The supervisors of the VHTs also carried out weekly mentorships to the VHTs for them to be able to know how to use these equipment's and supplies.

\subsubsection{Supervision}

Supervision conducted on a regular basis to carry out administrative tasks and to provide individual performance support (feedback, coaching, data-driven problem-solving). Regular supervision visit every 3 months that includes reviewing reports and monitoring data collected. Supervisors are trained in supportive supervision, have the technical skills to do service delivery observations, and have basic supervision tools (checklists) to aid them. Data is used for problem solving and coaching during supervision meetings. Supervisor visits community, makes home visits with $\mathrm{CHW}$, and provides skill coaching to $\mathrm{CHW}[1,6$, and 18]. Figure 4 (CHV supervision) shows that supervision moved from 0 score to the highest score of 3. The EAMNCH project implementation of the $\mathrm{ttC}$ model required that VHTs are actually mentored on a quarterly basis by their supervisors 
since $\mathrm{ttC}$ was a Behavioral Change communication (BCC) model that required timely counselling and the VHTs needed to take on the counselling skills and therefore the contribution that made this component score highly.

\subsubsection{Performance Evaluation}

This component focuses on the evaluation of the CHW to fairly assess their work during a set period of time. Most guidance encourages that this is done at least once a year and this evaluation should include individual performance including an assessment of service delivery based on documented supervisory feedback and an evaluation of coverage or monitoring data. Further still the community is supposed to be asked to provide feedback on CHW performance. There should therefore be clear rewards for good performance, and the community should play a role in providing awards. The $\mathrm{MoH}$ guidelines of 2010 have not given specific indicators for this kind of support to the VHTs. The policy provides for supervision and also encourages feedback to the CHWs and for those that have a good performance to be rewarded by making them peer supervisors of their counterparts. The guidelines also encourage the community to also reward the VHTs however no guidance is provided on how to reward, how frequent and the means of this reward neither is there guidance on the platform for reward. The project struggled with this program component as seen in figure 4 (CHW Performance Evaluation) at the onset of the project, the component scored
0 , and improved to 1.5 at the mid-term review but declined to 1. This is an indication that the projects contribution to this program component did not have any strategic guidance and therefore was not prioritized.

\subsubsection{Incentives}

A balanced incentive package includes financial incentives such as salary and bonuses and non-financial incentives such as training, recognition, certification, uniforms and even medicines for community support. In some instances this could include community incentives like offering of food during the harvest season, labor to the CHW during the gardening season or construction in the VHTs compound without pay, and recognition at major functions like weddings, traditional ceremonies and funerals. The guidance is that incentives are balanced, with both financial and nonfinancial provided, and are in line with expectations placed on $\mathrm{CHW}$, for example number and duration of visits to clients, workload, and services provided [3]. However incentives should be partially based on performance relevant to expectations and include advancement opportunities. The best performing CHWs are rewarded by offering them opportunities to join the formal education and be certified as nurses. The Ugandan guidelines of 2010 stipulate that the VHT should be given an equivalent of 10USD every time they are engaged in work, during a meeting, report submission. It also encourages partners to provide T-shirts, caps, gumboots, bag and umbrella.

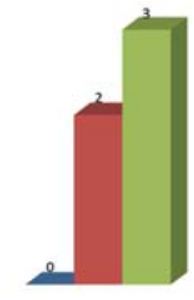

CHV Supervision

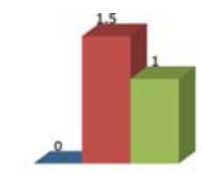

CHW Performance Evaluation

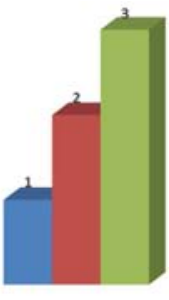

Incentives

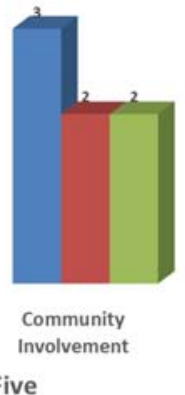

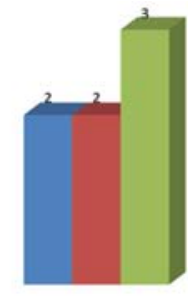

Referral System

- Yrone $\quad$ Yr Three $\quad$ Yr Five

Figure 4. Comparision of the Second 5 core components throughout the project cycle.

However there is no commitment from the $\mathrm{MoH}$ on a monthly mandatory incentive. Monitory incentive is tagged to a particular activity and non-financial is not that streamlined and therefore partners including this project offered them at their pleasure. From Figure 4 (incentives) shows that the project had a progressive improvement from baseline with a score of 1 progressing to a score of 2 and eventually contributing to a good practice score of 3 . Financially the VHTs had to submit a monthly $\mathrm{ttC}$ report and this earned them a 10USD and non-financially T-shirts, Umbrellas, Gumboots where provided to the VHTs. The project towards the closure introduced a more sustainable platform for motivation of the VHTs, the VHTs were clustered into groups of 10 and they were supported to come up with income generating concepts, they were supported to start and sustain this income generating activity. This included piggery, poultry, goat, and cattle rearing. Some decided to do brick making, shop keeping, cash box while others decided to open up large farm lands. This was thought to be more sustainable than cash and hence the score of 3 .

\subsubsection{Community Involvement}

This program component focuses on the role that the community plays in supporting $\mathrm{CHW}$, this includes supervising, offering incentives, and providing sincere feedback to the CHW. The community is supposed to play an active role in all support areas for $\mathrm{CHW}$ such as developing role, providing feedback, solving problems, providing incentives and helps to establish CHW as a leader in community therefore $\mathrm{CHW}$ is widely recognized and appreciated for providing service to community $[19,20]$. The Community leaders are supposed to have ongoing dialogue with $\mathrm{CHW}$ regarding health issues using data gathered by the CHW and the community interacts with supervisor during visits to provide feedback and solve problems. The Ugandan 
$\mathrm{MoH}$ guidelines of 2010 provide for the involvement of the community and community leadership in the VHT work, selection and apportioning area of coverage, agreeing on the roles and providing feedback to the VHTs. However the guidelines do not provide a deliberate mechanism for the feedback to be given, for example there is no VHT feedback events provided for by the policy guidelines and therefore any feedback given is an opportunity. Figure 4 (community involvement) shows that at the onset of the project, the process seemed to be a best practice with a score of 3, however as implementation went by, the score dropped to a 2. The drop was caused by the fact that it was agreed that the community had little or no interaction with the sub county Health assistant who supervisors the VHT. This is a true revelation since there was no provided framework for such deliberate meetings and engagements to happen.

\subsubsection{Referral System}

For an effective community referral system, there should be a process for determining when referral is needed, logistics plan for transport and payment to a health care facility when required, how referral is tracked and documented [16]. The CHW should be able to know when to refer clients through the identification of danger signs, and offer guidance on additional treatment needed. The CHW and community members are expected to know where the nearest referral facility is, usually have means for transport and have a functional logistics plan for emergencies. When a client is referred the process needs to include a standardized form and information flows back to $\mathrm{CHW}$ with a returned referral form. The Ugandan $\mathrm{MOH}$ guidance provides for every VHT to be answerable to the nearest health facility, should be accountable to the in charge of that health facility and should submit reports to the nearest health facility. This also includes referring emergencies and clients in ill health to the same health facility. The guidelines further provides for a counter referral form, the VHT initiates the referral process by handing the filled in referral form to the client, who hands it over to the health facility staff on arrival and after stabilization and treatment the Health facility staff provides a tear off portion back to the client to return to the VHT. The guidance also encourages the health facility staff to offer immediate support to this client who appears to the Health facility with the referral form from the VHT. Figure 4 (referral system) shows that right from inception the VHT has been facilitating effective referral with a score of 2 , and this progressed to a 3 by the close of the project. The implementation of the $\mathrm{MoH}$ recommendation here was a great contributor to this good practice.

\subsubsection{Professional Advancement}

This focuses on the possibility for growth, advancement, promotion and retirement for CHW including certification, increased responsibilities, and path to formal sector or change in role. Advancement is offered to CHWs who perform well and who express an interest in advancement if the opportunity exists [3]. Training opportunities are offered to CHWs to learn new skills to advance their roles and CHWs are aware of them. Advancement is intended to reward good performance or achievement and is based on a fair evaluation [21]. Although the Ugandan $\mathrm{MoH}$ guidance does not provide a clear path to formal advancement, there is a strong leadership provision, for example best performing VHTs are invited and scheduled to support at the Heath facility in triaging and providing Health education while clients are waiting. Further still the best performers are transformed into VHT parish supervisors and sub county VHT leaders. Figure 5 (advancement opportunity) shows that the project found this component at Zero and contributed to its progress to 2.5 and then dropped to 2. The basic contribution to this was mainly assigning the VHTs roles at the integrated community outreach sites, strengthening and accelerating the promotion of best performers to the role of supervisors and training them into special stepping stones experts.

\subsubsection{Documentation, Information Management}

This component focuses on how the CHWs document visits, how data flows from the $\mathrm{CHW}$ and gets integrated and utilised with in the health system and back to the community, and how it is used for service improvement at the community level. The component seeks to strengthen CHWs documentation of their visits consistently, Supervisors monitor quality of documents and provide help when needed and how the CHWs and communities work with supervisor to use data in problem solving at the community level [4]. The Ugandan $\mathrm{MoH}$ guidance about this is that the VHT updates the household register periodically after conducting the quality walks with in the catchment area, the VHT is supposed to be given a quarterly report that is submitted to the VHT parish supervisor, who then submit to the nearest health facility where the VHT is attached. It's at this level that the data is compiled and submitted to the Health centre iii, found at the sub-county level.

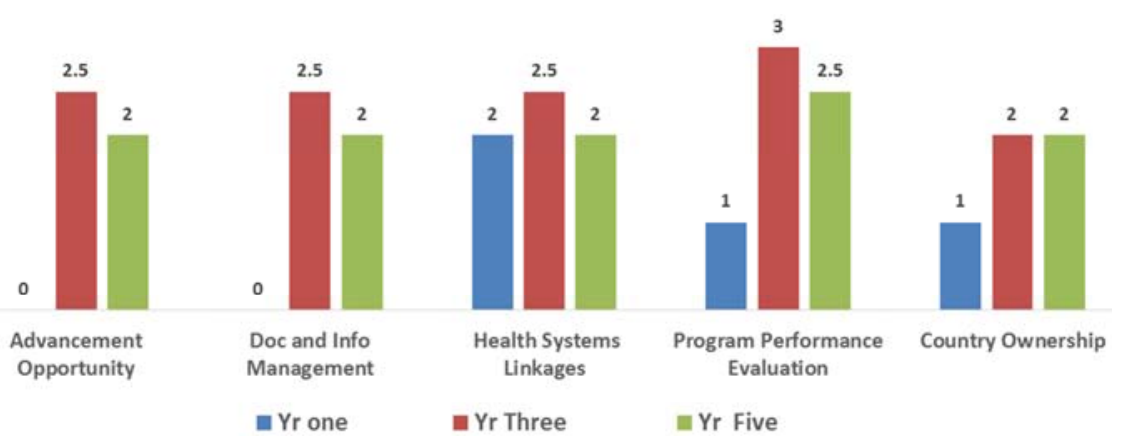

Figure 5. Comparision of the third 5 core components throughout the project cycle. 
This data from this point joins the health system through the Health management and information system, the path it takes to the district and then to the national level. The Health centre is further expected to convene a quarterly meeting with the VHTs to discuss among other things data, implication to the communities looking and focusing on issues that came out of the data as poor indicators. The VHT is supposed to take this feedback to the community for example if its poor latrine coverage, the VHT mobilizes the community to sink more latrines, if its poor ANC coverage, the VHTs then focus on that. Figure 5 (Doc and Info management) shows that at the onset of the project, the score was at 0 and it moved to a very good 2.5 improvement and finally reduced to 2 . This final score is a functional score meaning that the VHTs actually had the documents (household register and the quarterly reports) and that the data flow was followed well, the team also agreed that the VHTs reported on time and they received timely feedback from the supervisors, the supervisors also mentored the VHTs periodically, however one key challenge was identified, the Feedback mechanism to the community was not effective, the community was not deliberately given feedback and therefore community level action planning and passing of bylaws about health specific actions was not popular in this sites even when they were implementing ttC.

\subsubsection{Linkages to Health System}

Implementation of this program component focuses on CHWs and communities linkage to the larger health system. Health System is made of government, regions, districts, municipalities and individual health facilities that provide resources, finances and management to deliver health services to the population [7]. For it to be successfully implemented means that the Health system has comprehensive support mechanisms for all fundamental aspects of CHW program and that the initial and continuing training and on-site coaching supervision is the responsibility of the health system. Further still that the guidelines for supervision are clear; supervisors visit $\mathrm{CHW}$ for performance review, coaching, and problem solving and document results from this exercise. That the health system provides guidance on referral, and counter-referral [5]. That the health system has a mechanism for tracking and resupplying $\mathrm{CHWs}$, and that the health system has a clear incentives framework both monetary and non-monetary based on performance advancement. That $\mathrm{CHW}$ monitoring data are reviewed, analyzed, and included in health facility/ system reporting and national health monitoring information system [1, 3, 22 ]. Figure 5 (Health systems linkages) shows that the project found this component at a score of 2 , but caused an improvement to 2.5 but by the close of the project the score had gone back to 2 . This is a good practice and the system is actually functional. This is because all that is stated above as the ideal situation is the position of the district and the $\mathrm{MoH}$. All the capacity building follows a cascade model and is done by the district, reporting actually fits into the HIMS, the incentives given to the VHTs is by policy, and the referral system is streamlined however the fear of the scoring team was that the $\mathrm{MoH}$ and the district does little to avail the financial and logistical support to make these a reality. All financial and logistically support for this system to function is partner dependent and therefore cannot be a best practice.

\subsubsection{Program Performance Evaluation}

The implementation of this program component encourages the district and the project to perform a general program evaluation of performance against targets, focusing on the overall program objectives, setting and following through with indicators and this is carried out on a regular basis. The most realistic practice is facilitating an annual evaluation conducted of CHW activities that assesses CHW achievements in relation to program indicators and targets. The assessment should include an evaluation of the quality as well as the quantity of service delivery provided by CHWs, and the community is asked to provide feedback on CHW performance [23]. Health workers are asked to provide feedback based on data received from CHWs. Feedback is provided to CHWs on how they are performing in relation to program indicators and targets and against service delivery standards. The Ugandan $\mathrm{MoH}$ encourages districts to evaluate the VHTs, and there are agreed upon indicators for the performance of the VHTs and these trickle down to the variables that are included with in the HMIS on a monthly and quarterly basis. Figure 5 (program performance evaluation) presents an improvement from the score of 1 to 3 by the midterm but this then declines to a score of 2 . The project facilitated the annual evaluations for the VHT program using this CHW-AIM. A process that was involving the partners and the district, indicators where discussed and feedback to the community was given. However the programming team that participated in the scoring at the end of the project agreed that this is no longer going to be sustained and the district by this time had not shown any commitment financially to continuing this process. The district was commended for always making sure that they facilitate this process technically and included it in their annual work plan but there was no evidence of financial allocation for this intervention.

\subsubsection{Country Ownership}

The focus of this program component is the extent to which the $\mathrm{MoH}$ has integrated the $\mathrm{CHW}$ cadre in health systems planning specifically looking at the policies in place and how these policies are being implemented, budgeted for period and sustained financial support at all levels, evidently has plans and provides logistical support including supervision, and supplies to sustain $\mathrm{CHW}$ programs at a district, and national level. Therefore CHWs are actually recognized as part of the formal health system [3, 21,23]. Figure 5 (Country ownership) shows that at the time of the onset of the project the score was 1 but then improved to 2 but stagnated there until the project closed. It should be 
strongly noted that the Ugandan $\mathrm{MoH}$ has got one of the best policy framework as far as the VHTs are concerned, the staffing norms for attending to VHT work is strong from the national level to the district and the lower levels, annual work plans include VHT related activities however the challenge that kept this score at 2 is the inability to actually commit Financial resources for the teams to implement these activities. So there is visible reliance on partner support and in most cases the partners can only support action plan components that contribute to their desirables and or their geographical location thereby partially supporting the heath system.

\subsection{Overall Functionality Performance of the EAMNCH Project Against the District}

As highlighted in the methodology, the CHW-AIM was not only done in the project area but was done as part of a district wide process. All the sub-counties in the district had to be assessed, simply because the project contributed to the health systems but also needed to compare the status of the district to that of the project site. The district facilitated this process, and therefore made sure it's done in the entire district. As seen in Figure 6 below, generally the EAMNCH project site performed much better than the district. This could have been due to the fact that it was a project deliverable to implement the project specific action plan which was not the case for the other sub counties in the district. The other implementing partners further may not have had any financial muscle to implement the actions and therefore not being able to pull up the district scores to those of the EAMNCH project area. It's important to note that the district score also includes the 2 sub counties where the EAMNCH project was based.

\section{Lessons Learnt}

a) Involvement of the district health team is paramount this is because it brings a system's thinking in the whole process. When the district is involved in a self-appraisal of its own community health system, there are commitments done at that level to try and implement the action plans and therefore improve the community system together.

b) When the process is done with other implementing partners in the project site that have a VHT bias effects resources utilisation and prevents duplication of efforts. The funding going to the VHTs activities in a given project site are spent selectively since partners agree on what to focus on during the action planning process.

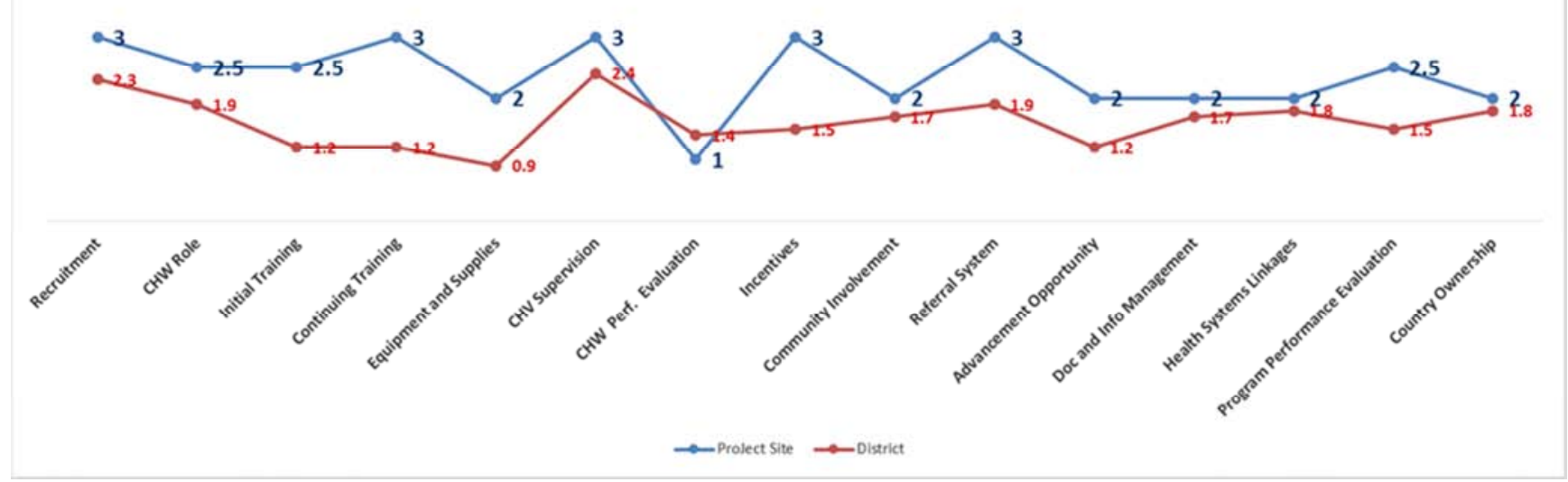

Figure 6. EAMNCH project site in comparision to Kitgum district program scores.

a. Involvement of VHTs, in the process went a long way in motivating them as they assessed their performance and were involved in the improvement plans.

b. Periodic utilisation of the tool at the district level gave the project a yardstick of performance as it's a comparision to other sub-counties where the project was never implemented.

\section{Challenges and or Missed Opportunities}

a) There was limited political involvement in the whole process from the first to the final year thus limited political commitments and therefore no district level priority setting around the value addition of this exercise.

b) Through the life cycle of the project, the district and sub county councils never made any financial contributions and therefore no opportunity to witness this process being financially supported by the District.

c) Even though other district partners participated and were willing to utilize the outcomes of this annual exercise, there was no actual adaptation of the practice by these partners in other project sites within the district. None of the district partners adapted this in their annual work plan.

d) The CHW-AIM fifth year action plan may never be implemented, and the community Health workers in the project site may end up being abandoned since the district had not shown any financial commitment to the continuity of the interventions. 


\section{Discussion and Recommendations}

\subsection{Discussion}

The EAMNCH 5 year life cycle and the lessons captured during this project implementation period, it's important to emphasis that for CHW programs to perform effectively, it is vital to lay proper emphasis on training and supervision. The project supported a MNCH household model that needed the VHT to be able to pass on the information and facilitate actions through a negotiation with the mother or father and for this to happen meant that the VHTs should have been trained and there needed to be continuously on job mentorship and supervision. This critically went a long way to ensure that the project actually promoted the actions for good MNCH outcomes at household level.

CHW programs should regulate a clear selection and deployment procedure. Ideally, the community should be engaged in planning, selecting, implementing, and monitoring, which reinforce the appointment of those who have been entrusted the VHT role by the same community. The EAMNCH experience clearly points out that the community seemed to be at the receiving end and did little in holding the VHTs accountable. For example the community never focused on how to actually motivate the VHTs for the work they have been doing to make sure that the survival of all pregnant mother and children below two years is uplifted. The government should take responsibility for making a transparent system for selection and deployment and further quality assurance of the regulated system. When scaling up a CHW program, decision makers should consider how to link them up with the wider health system [24], but also create mechanisms that generate appreciation for the VHT work from the wider community.

Prior experiences have documented that low interest use by the government, inconsistent remuneration, inadequate staff and supplies are key factors that negatively impact the $\mathrm{CHW}$ program. These factors can be alleviated through certified training programs and supportive supervision, along with offering other incentives (financial and nonfinancial) to keep CHWs satisfied and motivated to perform their duties well. Furthermore, efforts geared toward standardizing training and certification for CHW programs, could further provide a career pathway and enable them to effectively contribute to their communities health [25]. The major challenge in this EAMNCH implementation was the question of $\mathrm{ttC}$ is a world Vision model and all the 255 VHTs were trained in it, together with the supplies that come with the model, all VHTs sent in reports on a timely manner however, with the exit of World Vision from the project site, whoever comes in to carry on the $\mathrm{MNCH}$ work, will they pick up from where World Vision stopped or will there be a new MNCH curriculum with totally new reporting tools and implementation mechanism that will further need the same rigorous process to make VHTs get to implement the new model. Then who will be providing the 10USDs for meetings and reporting if this project area does not get a partner to support any time after the closure.
The VHT programs should be coherently inserted in the wider health system, and CHWs should be explicitly included within the human resources for health strategic planning first at country and local level. The Ugandan health care structure actually provides for the human resources, including paid supervisors from the $\mathrm{MoH}$ down to the sub county level however there has been no indication to include human resources remunerations at the village level. While CHWs may not replace the need for sophisticated and quality health care delivery through highly skilled health care workers, they could play an important role in increasing access to health care and services, and in turn, improved health outcomes, as an effective link between the community and the formal health system, and as a critical component in the efforts for a wider approach that takes into account social and environmental determinants of health [25].

\subsection{Recommendations}

a) The District of Kitgum needs to sustain the data flow to the HMIS but also implement the feedback mechanism of this information to the community to help in trucking VHTs performance for proper service improvement, celebrations and ownership by the community.

b) $\mathrm{MoH} / \mathrm{District}$ should determine and follow up aspect of equipping VHTs with necessary Logistics to improve their performance.

c) The District and the Health Facilities should use this VHT Assessment tool periodically to identify areas of weakness and make improvements accordingly.

d) There is need for consistent, proper and quality documentation of the VHTs work by VHTs themselves, the supervisors, health facilities, and District working together with the development partners sustain this action plan.

\section{Conclusion}

Kitgum's poor health indicators at the onset of the EAMNCH project, were partly tackled through constant dialogue with the community members using VHTs. VHTs helped bridge the gap that exists between the un-served households and the formal health system. VHTs were established by the $\mathrm{MoH}$ to empower communities to take part in the decisions that affect their health; mobilize communities for health programs, and strengthen the delivery of health services at house-hold level. It was therefore important to institute a VHT functionality improvement implementation framework and use the CHW-AIM to improve health service availability and access to households in the project site. World vision Uganda through its EAMCH project in Kitgum district supported the establishment and capacity building of VHTs in the operational areas with the MOH's guidance. VHTs have been operationalized, provided training on programmatic areas with the basic/core training required of VHTs using the MOH VHT training manual and tried to support the other components that promote the functionality of the VHT Program. This has largely contributed to the results highlighted above. 


\section{Acknowledgement}

The East African $\mathrm{MNCH}$ project was implemented with Donations from the Australian Government, Department of Foreign Affairs and Trade as part of the Australia Africa Community Engagement Scheme (AACES) through the World Vision Australia office. Funding was utilised by World Vision Uganda in partnership with the Kitgum District Local government from 2011 to 2016

\section{References}

[1] WHO, 2007, Community health workers: What do we know about them?:

http://www.who.int/hrh/documents/community_health_worker s.pdfMinistry of Health Uganda, 2010, Village.

[2] Health Team. A handbook to improve health in communities. https://www.k4health.org/sites/default/files/VHT\%20BOOK.pdf.

[3] URC, 2011, Community Health Worker Assessment and Improvement Matrix (CHW AIM): A Toolkit for Improving CHW Programs and Services: http://www.urcchs.com/sites/default/files/Community $\% 20$ Health\%20Worker $\% 20$ Assessment $\% 20$ and $\% 20$ Improvement $\% 20$ Matrix.pdf.

[4] World Vision Uganda, 2014, Improving Maternal Newborn and Child Health through Village Health Teams in Uganda: A VHT AIM Functionality Assessment Report.

[5] WHO, 2006, The cost-effectiveness of close-to-community health programmes:

http://www.who.int/workforcealliance/knowledge/themes/com munity/en/.

[6] MoH, Uganda, 2015, NATIONAL VILLAGE HEALTH TEAMS (VHT) ASSESSMENT IN UGANDA http://library.health.go.ug/publications/service-deliverypublic-health/health-education/national-village-health-teams.

[7] World Vision Uganda, 2011, East Africa Maternal, Newborn and Child Health Project (EAMNCH) Project Design document

[8] World Vision International, Global Health and Nutrition, 2010, 7-11 Field start up guide: http://www.wvi.org/sites/default/files/WV_FieldGuide_FINA L_2.pdf.

[9] Mitchell H. Katz, MD, 2012, The Value of Community Health Workers. Comment on Community Health Workers Combat Readmission.http://jamanetwork.com/journals/jamainternalme dicine/fullarticle/1389235.

[10] Auma Jane-Frances Nyasuna 2011, The Ugandan Government's commitment to the Right to Health: a focus on Village Health Teams (VHT) and the rural poor.

[11] Rornald Kananura, 2012, Role of Village Health Teams (VHTs) in Public Health-Uganda, https://apha.confex.com/apha/140am/webprogram/Paper26813 2.html.

[12] Dr. Henry Katamba, 2013, iCCM in Uganda. Background and Process. http://www.malariaconsortium.org/userfiles/5-ICCMin-uganda-background-and-process.pdf.

[13] One Million Health worker campaign, 2014, The Current State of CHW Training Programs in Sub-Saharan Africa and South Asia: What We Know, What We Don't Know, and What We Need to Do.

http://1millionhealthworkers.org/files/2013/01/1mCHW_mPo wering_LitReview_Formatted.compressed.pdf.

[14] Global Workforce alliance, 2011, Community Health Worker Assessment and Improvement Matrix (CHW AIM): A Toolkit for Improving Community Health Worker Programs and Services. Tool for conducting assessment and improvement for community health worker practice.

http://www.who.int/workforcealliance/knowledge/toolkit/54/en/.

[15] Babughirana Geoffrey, Muhirwe Barungi Lorna, Kimurahebwe Clare. Village Health Team Functionality in Uganda: Implications forCommunity System Effectiveness. Science Journal of Public Health. Vol. 4, No. 2, 2016, pp. 117 126. doi: 10.11648/j.sjph.20160402.16.

[16] World Vision International, 2014, TIMED AND TARGETED COUNSELLING FOR HEALTH AND NUTRITION. A TOOLKIT FOR PROGRAMME PLANNERS. http://chwcentral.org/sites/default/files/TTC\%20Toolkit\%20fo r\%20Programme\%20Planners.pdf.

[17] World Vision Uganda, 2014 Implementation guideline for the TIMED AND TARGETED COUNSELLING.

[18] Lauren Crigler, Jessica Gergen, and Henry Perry, 2013, Supervision of Community Health Workers. http://www.mchip.net/sites/default/files/mchipfiles/09_CHW_ Supervision.pdf.

[19] Kimbugwe et al. 2014, Challenges Faced by Village Health Teams (VHTs) in Amuru, Gulu and Pader Districts in Northern Uganda,

https://www.ncbi.nlm.nih.gov/pmc/articles/PMC4542049/pdf/ nihms-714126.pdf.

[20] MINISTRY OF HEALTH, REPUBLIC OF UGANDA, 2016, VHT / Community Health Extension Workers. http://health.go.ug/community-health-departments/vhtcommunity-health-extension-workers.

[21] Illinois Career Information System, 2015, Community Health Workers Advancement Opportunities. https://apps.il-worknet.com/cis/clusters/OccupationDetails/100565?parentId $=1110$ $00 \&$ section $=$ advancement\&section Title $=$ Advancement $\% 200 p$ portunities.

[22] WHO, 2012, Bulletin of the World Health Organization. Deployment of community health workers across rural subSaharan Africa: financial considerations and operational assumptions. http://www.who.int/bulletin/volumes/91/4/12109660/en/.

[23] Maryse Catelijne Kok, 2015, PERFORMANCE OF COMMUNITY HEALTH WORKERS. Optimizing the benefi ts of their unique position between communities and the health sector. http://213ou636sh0ptphd141 fqeil.wpengine.netdnacdn.com/health/wpcontent/uploads/publications/5641fbb74cc7f_Kok\%202015\% 20Performance $\% 20 \mathrm{of} \% 20 \mathrm{CHWs}$.pdf.

[24] Bjerregaard D, Crigler L. 2012. CHW Regional Meeting. Addis Ababa,Ethiopia, June 19-21, 2012. Proceedings. Published by the USAID Health Care Improvement Project.

[25] Ministry of Health (MOH) Uganda. 2009b. "Situation Analysis, Village Health Teams Uganda. 